\title{
Humidity is a consistent climatic factor contributing to SARS-CoV-2 transmission
}

\author{
Michael Ward ${ }^{1}$, Shuang Xiao ${ }^{2}$, and Zhijie Zhang ${ }^{3}$ \\ ${ }^{1}$ University of Sydney \\ ${ }^{2}$ Fudan University \\ ${ }^{3}$ Affiliation not available
}

July 7,2020

\begin{abstract}
There is growing evidence that climatic factors could influence the evolution of the current COVID-19 pandemic. Here, we build on this evidence base, focusing on the southern hemisphere summer and autumn period. The relationship between climatic factors and COVID-19 cases in New South Wales, Australia was investigated during both the exponential and declining phases of the epidemic in 2020, and in different regions. Increased relative humidity was associated with decreased cases in both epidemic phases, and a consistent negative relationship was found between relative humidity and cases. Overall, a decrease in relative humidity of $1 \%$ was associated with an increase in cases of 7-8\%. Overall, we found no relationship with between cases and temperature, rainfall or wind speed. Information generated in this study confirms humidity as a driver of SARS-CoV-2 transmission.
\end{abstract}

\section{Introduction}

The global spread of severe acute respiratory coronavirus 2 (SARS-CoV-2), causing the novel coronavirus disease (COVID-19) pandemic, has been linked to climatic factors. This has a biological basis. The spread of SARS-CoV-2 among people is predominantly via respiratory droplets and aerosols, as well as fomites (Cai et al., 2020) and possibly fecal-oral (Yeo et al., 2020). Temperature and relative humidity can affect coronavirus transmission (Casanova et al., 2010) through virus survival (at lower temperatures coronaviruses survive longer) and the length of time infectious respiratory matter stays suspended in the air (at lower humidity more material stay suspended for longer) (Casanova et al., 2010; Chan et al., 2011; Guionie et al., 2013).

In previous observational research, a negative relationship between relative humidity and SARS cases has been found (Cai et al., 2007; Tan et al., 2005), and a similar negative relationship with Middle East respiratory syndrome coronavirus (MERS-CoV) cases has been described (Gardner et al., 2019; Altamimi and Ahmed, 2020). However, the relationship with temperature is inconsistent: a positive relationship has been described for SARS (Gardner et al., 2019) and MERS-CoV (Altamimi and Ahmed, 2020), but a negative relationship has also been observed for MERS-CoV (Gardner et al., 2019). Specifically for SARS-CoV-2, a negative relationship between COVID-19 cases in China and temperature and humidity has recently been described (Qi et al., 2020), and more recently in the state of New South Wales (NSW), Australia we described a significant negative association between COVID-19 cases during the initial exponential phase of the epidemic and relative humidity (Ward et al., 2020). In the current study we extend this research to examine the effect of a greater number of climatic factors on the occurrence of COVID-19 cases during both exponential and descending phases of the epidemic, and investigate whether there are regional and temporal differences in this relationship. This knowledge is needed to guide public health interventions to successfully control the spread of SARS-CoV-2. 


\section{Methods}

Case reports in NSW, Australia from the beginning of the epidemic in January to the end of May 2020 were accessed. ${ }^{1}$ Those whose infection source was determined to be locally-acquired, and whose postcode of residence was reported, were included. A daily time-series of cases was created, from which separate series preceding and following the epidemic peak (31 March) and for individual NSW public health units (PHUs, see Figure 1A) were created. Based on the reported postcode the closest weather observation station was identified. ${ }^{2}$ Daily observations of the following factors were downloaded: rainfall $(\mathrm{mm})$ and temperature $\left({ }^{\circ} \mathrm{C}\right)$, relative humidity $(\%)$ and wind speed $(\mathrm{m} / \mathrm{s})$ recorded at $9 \mathrm{am}$ and at $3 \mathrm{pm} .{ }^{2}$ The mean values for each day were estimated to create time-series of weather data. Additional series of daily differences between 9am and $3 \mathrm{pm}$ temperature, relative humidity and wind speed were created. Thus, 10 predictor time-series were created for modelling.

The data was analysed based on the exponential and descending phases of the epidemic overall, and for 6 PHUs (those PHUs reporting $<100$ cases were excluded), to determine the effect of epidemic phases and locations on the association between climatic factors and case reports. Thus, 14 separate time-series analyses were performed.

A PHU-average Spearman correlation coefficient matrix was first calculated to avoid multicollinearity among variables. Then, a univariate generalized additional model (GAM) was fit and variables with $P$ value $<0.1$ in univariate analysis in all the PHUs were selected for multivariate analysis. A standard two-stage approach was then applied to evaluate the PHU-specific and NSW-average associations between short-term exposure to climate factors and cases. In the first stage, a quasi-Poisson GAM was used to estimate the association between PHU-specific climate factors and the daily count of cases. A 14-day exponential moving average (EMA) was used to represent the effects of climate factors. Natural splines of time were included to control short-term temporal trend; its optimal degrees of freedom ( $d f$ ) was chosen based on Quasi AIC (QAIC). In the second stage, a meta regression model with random effects were used to obtain NSW-average risk estimate of meteorological factors on cases. To estimate the overall relationship, exposure-response curves were plotted using the GAM with natural spline's knot setting at its median $(d f=2)$. A sensitivity analysis was performed by modifying the EMA (14 days to 13 or 15 days), and changing $d f$ for natural splines of time (3 to 2 or 4). R4.0.1 software (R Foundation for Statistical Computing, Vienna, Austria) was used to perform all analyses.

\section{Results}

The first COVID-19 case in which infection was locally acquired was notified on 26 February $2020 .{ }^{1}$ Between 26 February and 31 May, 1203 locally-acquired cases with a residence postcode were notified. Cases were reported from 11 PHUs (range 3-357; Fig 1A); 6 of these reported $>100$ cases, and climatic data was acquired from 27 weather observation stations within these PHUs. ${ }^{2}$

Based on correlation coefficients, 3pm temperature and relative humidity, and temperature and relative humidity range were excluded from univariate modelling (Fig 1B). Overall, only 9am temperature (range 8.05-26.6) and 9am relative humidity (35-100\%) entered the final mode (Fig 1C). Mean temperature range was higher during the exponential epidemic phase than the descending epidemic phase, whereas the reverse occurred for relative humidity range (Table 1 ).

Overall, we observed a negative association between COVID-19 cases and relative humidity in both epidemic phases (Table 2), but no association with temperature. A $1 \%$ decrease in relative humidity was associated with a $7.7 \%$ (95\% CI: $0.04-14.8 \%)$ and $6.8 \%$ (95\% CI: $0.4 \%-12.2 \%)$ increase in the pooled estimate of daily counts of COVID-19 in the two epidemic phases, respectively. Heterogeneous effects across PHUs were obvious: a significant positive association between temperature and cases in South Eastern Sydney PHU during the descending epidemic phase (Figure 2A\&C), whereas a significant negative association between humidity and cases in Sydney PHU during the exponential epidemic phase (Figure 2B\&D), were noted. However, the association between humidity and cases was consistently negative in the epidemic phase- and location-specific analyses, except for South Western Sydney PHU in the exponential epidemic phase, and 
Western Sydney PHU in the descending epidemic phase (Table 2 \& Fig 2). The overall exposure-response curves showed that the negative association between cases and relative humidity was more pronounced above $79 \%$ and $75 \%$ relative humidity in the exponential and descending epidemic phases, respectively (Appendix 1). The sensitivity analysis indicated that the associations between cases and relative humidity were robust (Appendix 2).

\section{Discussion}

We found that throughout the epidemic of COVID-19 in NSW, Australia - both during the exponential and descending phases of the epidemic - there was a consistent negative relationship between relative humidity and case occurrence: a $1 \%$ decrease in relative humidity was predicted to increase cases about 7-8\%, with a more pronounced effect at a relative humidity $<75-79 \%$. In almost all PHUs this negative relationship between relative humidity and cases was found.

Given that SARS-CoV-2 transmission is thought to be primarily via the respiratory route (Cai et al., 2020), and that coronaviruses are known to be susceptible in the environment (Casanova et al., 2010), the finding of an association with relative humidity is expected. This association might occur via the effect on respiratory aerosols and therefore infectious material remaining airborne for longer; or it could be a more direct effect on the survivability of the virus in the environment. The lack of a consistent association between temperature and COVID-19 cases in this and other studies (Qi et al., 2020) - as well as for SARS and MERS-CoV cases (Tan et al., 2005; Gardner et al., 2019; Altamimi and Ahmed, 2020) - suggests that it is the former that influences SARS-CoV-2 transmission. This raises an interesting question, and one with potentially profound importance for public health: could increasing relative humidity contribute to a reduction in SARCoV-2 infections when infectious individuals mix with susceptible individuals? In the current study - as with other studies conducted to date on SARS, SARS-CoV-2 and MERS-CoV - we used data collected from meteorological recording stations under the assumption that either cases were infected in an outdoors setting, or that ambient outdoors weather conditions are a proxy for the indoors environment (if that is where most infections occur). Measuring the indoors environment is not possible when retrospectively analyzing hundreds of disease cases that have occurred in an epidemic across an entire country or state. The conduct of controlled studies of the relationship between COVID-19 cases and factors such as relative humidity is challenging.

It is important to highlight that COVID-19 cases used in this study occurred predominantly during the autumn season in southern hemisphere. In contrast, most COVID-19 cases in northern hemisphere have been reported during the winter and spring seasons. Despite the seasons being diametrically opposed, the negative relationship between humidity and cases we observed in the Australian autumn is consistent with that observed in the Chinese winter (Qi et al., 2020). Combined with evidence from studies in the northern hemisphere, the influence of relative humidity on COVID-19 incidence was found to be always negative in different regions, suggesting that the relationship could be universal: humidity is more sensitive to COVID19 transmission and periods of lower humidity might forecast spikes in SARS-CoV-2 transmission. In the absence of a vaccine, such observations allow the more timely, efficient and effective deployment of public health interventions.

\section{Acknowledgments}

NSW Ministry of Health is thanked for freely making available COVID-19 case notification data.

\section{Conflict of interest and funding}

Not applicable

\section{Footnotes}

1 NSW Government. Data.NSW: COVID-19 cases by notification date, location. https://data.nsw.gov.au/dataset/nsw-covid-19-tests-by-location-and-result. Accessed 3 June 2020. 
2 Australian Government. New South Wales weather observation stations. http://www.bom.gov.au/nsw/observations/map.shtml. Accessed 6 June 2020.

\section{References}

Altamimi A, Ahmed AE. Climate factors and incidence of Middle East respiratory syndrome coronavirus. J. Infect. Public Health 2020; https://doi.org/10.1016/j.jiph.2019.11.011

Cai J, Sun W, Huang J, Gamber M, Wu J, He G. Indirect virus transmission in cluster of COVID-19 cases, Wenzhou, China, 2020. Emerg. Infect. Dis. 2020; https://doi.org/10.3201/eid2606.200412

Cai QC, Lu J, Xu QF, Guo Q, Xu DZ, Sun QW, et al. Influence of meteorological factors and air pollution on the outbreak of severe acute respiratory syndrome. Public Health 2007;121:258-265.

Casanova LM, Jeon S, Rutala WA, Weber DJ, Sobsey MD. Effects of air temperature and RH on coronavirus survival on surfaces. Appl. Environ. Microbiol. 2010;76:2712-2717.

Chan KH, Peiris JS, Lam SY, Poon LL, Yuen KY, Seto WH. The effects of temperature and relative humidity on the viability of the SARS coronavirus. Adv. Virol. 2011;2011:734690.

Gardner EG, Kelton D, Poljak Z, Van Kerkhove M, von Dobschuetz S, Greer AL A case-crossover analysis of the impact of weather on primary cases of Middle East respiratory syndrome. BMC Infect. Dis. 2019;19:113.

Guionie O, Courtillon C, Allee C, Maurel S, Queguiner M, Eterradossi N. An experimental study of the survival of turkey coronavirus at room temperature and +4 degrees C. Avian Pathol. 2013;42:248-252.

Qi H, Xiao S, Shi R, Ward MP, Chen Y, Tu W, et al. COVID-19 transmission in Mainland China is associated with temperature and humidity: a time-series analysis. Sci. Total Environ. 2020;728:138778

Tan JG, Mu LN, Huang JX, Yu SZ, Chen BH, Yin J. An initial investigation of the association between the SARS outbreak and weather: with the view of the environmental temperature and its variation. J Epidemiol Community Health 2005;59:186-192.

Ward MP, Xiao S, Zhang Z. The Role of Climate During the COVID-19 epidemic in New South Wales, Australia. Transbound Emerging Dis 2020; https://doi.org/10.1111/tbed.13631

Yeo C, Kaushal S, Yeo D. Enteric involvement of coronaviruses: is faecal-oral transmission of SARS-CoV-2 possible? Lancet Gastroenterol. Hepatol. 2020;5:P335-337.

\section{Hosted file}

COVID_NSW_TBED_tab1.docx available at https://authorea.com/users/318560/articles/467225humidity-is-a-consistent-climatic-factor-contributing-to-sars-cov-2-transmission

\section{Hosted file}

COVID_NSW_TBED_tab2.docx available at https://authorea.com/users/318560/articles/467225humidity-is-a-consistent-climatic-factor-contributing-to-sars-cov-2-transmission

\section{Hosted file}

COVID_NSW_TBED_fig1.docx available at https://authorea.com/users/318560/articles/467225humidity-is-a-consistent-climatic-factor-contributing-to-sars-cov-2-transmission

\section{Hosted file}

COVID_NSW_TBED_fig2.docx available at https://authorea.com/users/318560/articles/467225humidity-is-a-consistent-climatic-factor-contributing-to-sars-cov-2-transmission 